\title{
Numerical Solution of Casson Fluid Flow under Viscous Dissipation and Radiation Phenomenon
}

\author{
Naveed Akbar ${ }^{1}$, Sardar Muhammad Hussain ${ }^{2 *}$, Riaz Ullah Khan ${ }^{3}$ \\ ${ }^{1}$ School of Mathematics and Statistics, Sichuan University of Science and Engineering, Yibin, China \\ ${ }^{2}$ Department of Mathematical Sciences, Balochistan University of Information Technology, Engineering and Management \\ Sciences (BUITEMS), Quetta, Pakistan \\ ${ }^{3}$ School of Life Science and Engineering, University of Electronic Science and Technology of China, Chengdu, China \\ Email: navidakbar109@yahoo.com, *smhussain01@gmail.com, riazkhan@uestc.edu.cn
}

How to cite this paper: Akbar, N., Hussain, S.M. and Khan, R.U. (2022) Numerical Solution of Casson Fluid Flow under Viscous Dissipation and Radiation Phenomenon. Journal of Applied Mathematics and Physics, 10, 475-490.

https://doi.org/10.4236/jamp.2022.102036

Received: December 29, 2021

Accepted: February 21, 2022

Published: February 24, 2022

Copyright () 2022 by author(s) and Scientific Research Publishing Inc. This work is licensed under the Creative Commons Attribution International License (CC BY 4.0).

http://creativecommons.org/licenses/by/4.0/

\begin{abstract}
This article numerically investigates the $2 \mathrm{D}$, steady, laminar, incompressible fluid flow, mass and heat transfer of a non-Newtonian fluid model induced by stretching surface. A Casson fluid model is considered to study the nonNewtonian behavior of the flowing fluid. The magnetic field and a porous medium are considered in the flow momentum, whereas the viscous dissipation is also taken into account in the energy transport phenomena. To see the fluid concentration, the concentration equation is used. Furthermore, the Nusselt number coefficient and skin friction are modified with the addition of nonlinear stretching and radiation parameters. With the similarity transformation, the nonlinear governing partial differential equations are transformed into a system of ordinary differential equations and then numerically solved using a fourth-order Runge-Kutta scheme with the shooting method. The relevant parameters of interest are interpreted for graphical results. The results illustrate that the fluid energy increases effectively with an increase in the Eckert number, radiation parameter, and heat source parameter, while it decreases by increasing the Prandtl number and heat sink parameter. Both the wall skin friction and the wall Nusselt number coefficient decelerate with an increase in the Casson parameter.
\end{abstract}

\section{Keywords}

Casson Fluid, Porous Medium, Runge-Kutta Scheme, Shooting Method, Prandtl Number, Nusselt Number Coefficient

\section{Introduction}

Fluid dynamics is an important sub-discipline of fluid mechanics that describes the progression of fluids. Hydrodynamics and aerodynamics are the two major 
areas of fluid dynamics. It has many fascinating applications, like calculating stresses and moments on airplanes, determining the mass flow rate of petroleum through pipelines, forecasting weather patterns, and modeling fission bomb explosions, among other things. The three basic axioms of fluid flow are the conservation laws: 1) Momentum conservation, 2) Energy conservation, and 3) Mass conservation. Fluid flow piques the curiosity of scientists and engineers. This is because the forces it generates are of considerable interest to the engineering sector. A few of these fascinating applications are jet propulsion, aerofoil design, wind turbines, and hydraulic brakes. Fluids are mainly categorized into Newtonian and non-Newtonian fluid models. Newtonian fluids have a linear relationship between stress and strain. On the other hand, non-Newtonian fluids have a nonlinear stress-strain relationship. Both of these fluid categories are of great importance in our daily lives.

Non-Newtonian fluids have gained a lot of attention in the past decades because of their vast applications in various engineering fields (such as crude oil processing, plastic material development, and syrup medication research). In this work, we are using the Casson fluid model as a non-Newtonian fluid. There are several other non-Newtonian fluid models, but the Casson fluid is particularly important due to its unique characteristics. For example, Casson fluid behaves like an elastic solid, and the constitutive equation includes a yield shear stress for this fluid. This type of transport mechanism can be found in a variety of chemical and mechanical engineering disciplines as well as in food processing. Casson's fluid rheological model was initially given by Casson [1]. The Casson model establishes a plastic fluid model that exhibits shear thinning, yield stress, and large shear viscosity. Later on, Subba Rao et al. [2] discovered that for a very large wall shear stress, the Casson fluid model can be reduced to a Newtonian fluid. Bird et al. [3] analyzed the rheology and fluid flow behavior of visco-elastic materials. Mukhopadhyay studied the heat transport phenomena of Casson fluid over a non-linearly stretching surface [4]. Animasaun et al. [5] studied the transport mechanism of Casson fluid under the impact of an external heat source using an analytical approach. Nawaz et al. [6] and Awais et al. [7] had made significant contributions to the advantageous domain of Casson fluid.

In the modern era, a magnetic field is enforced on the fluid flowing in a manner to guide the flow. In the presence of a magnetic field, the study of electrically conducting fluid is characterized by magnetohydrodynamics (MHD). The concept of electrically conducting fluid was originally proposed by Hannes Alfvén [8]. K. Anantha Kumar [9] studied the effect of thermal radiation on MHD Casson fluid flow over an exponentially stretching curved sheet and observed how the temperature field improves with radiation, temperature-dependent thermal conductivity, and irregular heat parameters. Hayat et al. [10] and Hussain et al. [11] investigated MHD transport analysis along with viscous dissipation and Joule heating. Dero et al. [12] obtained triple solutions during the investigation of micropolar fluid with thermal radiation effect.

Thermal radiation has a significant impact on the fluid system's velocity and 
temperature transport. The transport mechanism has piqued the interest of scientists and engineers due to its numerous applications. C. Sumalatha and S. Bandari [13] carried out research on the flow over a non-linearly stretching sheet of Casson fluid with the effects of radiation and heat source/sink. Rudraswamy et al. [14] investigated the Jeffery nanofluid flow while taking Soret and Dufour into account. Kumar et al. [15] designed a mathematical model for a three-dimensional Jeffery fluid subjected to radiation and viscous dissipation. Rudraswamy et al. [16] studied the effect of Joule heating on a three-dimensional fluid flow. Sheikholeslami et al. [17] numerically compute the effects of an externally enforced magnetic field induced by stretching surface. However, in order to study heat transfer phenomena, many researchers have included nonlinear thermal radiation in their models. More interesting investigations on heat transfer on natural convection, MHD and porous medium can be seen [18]-[23].

In the current work, we numerically investigated the importance of Casson fluid flow and heat transfer mechanism in non-Newtonian fluid flow over a sheet. In order to determine the fluid momentum behavior, a magnetic field and a porous medium are considered in the linear momentum equation. The energy transfer analysis is also investigated under the impact of thermal radiation, heat source/sink, and viscous dissipation phenomena. Furthermore, the fluid concentration is observed via the concentration equation. The $x$-axis is extended non-linearly, whereas the $y$-axis is set normal to it.

\section{Mathematical Formulation}

Consider a 2D, steady and incompressible flow of a Casson fluid passing through a porous stretching surface at $y=0$. The flow is limited to $y>0$, whereas the $x$ and $y$-axes are taken along and normal to the surface respectively. Figure 1 displays the geometrical view of fluid flow with the considered coordinate system and boundary-layer conditions.

The rheological equation of state for anisotropic and incompressible Casson fluid flow is

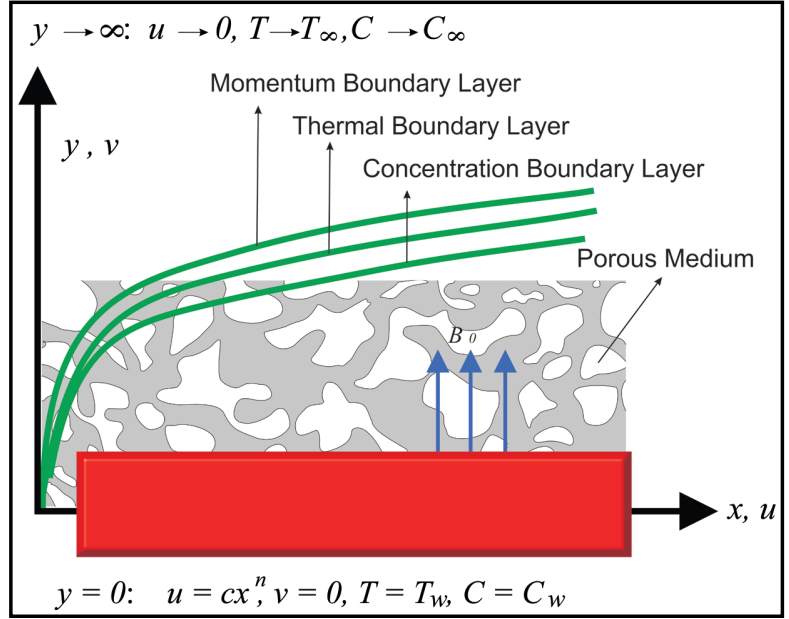

Figure 1. Geometrical view of the physical model. 


$$
\tau_{i j}= \begin{cases}2\left(\mu_{B}+p_{y} / \sqrt{2 \pi}\right) e_{i j} & \text { for } \pi>\pi_{a} \\ 2\left(\mu_{B}+p_{y} / \sqrt{2 \pi}\right) e_{i j} & \text { for } \pi<\pi_{a}\end{cases}
$$

where $\pi=e_{i j} e_{i j}$. In this work, we assume that $e_{i j}$ is the $(i, j)^{\text {th }}$ component of the deformation rate, $\pi$ is the product of the component of the deformation rate, $p_{y}$ is the yield stress of the fluid, $\pi_{a}$ is the critical value of the product based on the non-Newtonian model and $\mu_{B}$ is the plastic dynamic viscosity of the non-Newtonian fluid [24] [25] [26]. Furthermore, the set of partial differential equations, known as boundary layer equations or Prandtl equations, obtained using the well-known boundary layer approximations may take the form

$$
\begin{gathered}
\frac{\partial u}{\partial x}+\frac{\partial v}{\partial y}=0 \\
u \frac{\partial u}{\partial x}+v \frac{\partial u}{\partial y}=v\left(1+\frac{1}{\beta}\right) \frac{\partial^{2} u}{\partial y^{2}}-\frac{\sigma}{\rho} B_{0}^{2} u-\frac{v}{k^{*}} u \\
u \frac{\partial T}{\partial x}+v \frac{\partial T}{\partial y}=\kappa \frac{\partial^{2} T}{\partial y^{2}}-\frac{1}{\rho c_{p}} \frac{\partial q_{r}}{\partial y}+\frac{Q_{0}}{\rho c_{p}}\left(T-T_{\infty}\right)+\frac{v}{c_{p}}\left(1+\frac{1}{\beta}\right) \frac{\partial^{2} u}{\partial y^{2}},
\end{gathered}
$$

and

$$
u \frac{\partial C}{\partial x}+v \frac{\partial C}{\partial y}=D_{B} \frac{\partial^{2} C}{\partial y^{2}} .
$$

The above set of equations has been extensively used for various fluid flows to compute physical quantities like heat-transfer, skin friction coefficient, as presented [27] [28] [29]. Here $u, v$ represent the velocity components in $x$ and $y$ directions, respectively, while $v$ represents the kinematic viscosity, $\rho$ is the density, $\beta=\mu_{B} \sqrt{2 \pi_{c} / p_{y}}$ is the Casson fluid parameter, $\kappa$ is the thermal diffusivity, $c_{p}$ is the specific heat, $k^{*}$ is the fluid medium's permeability, $C_{w}$ is the concentration of fluid at surface, and $C_{\infty}$ is the concentration in the free stream. The appropriate boundary conditions are also determined by

$$
u=c x^{n}, \quad v=0, \quad T_{w}=T, \quad C=C_{w} \quad \text { at } y=0
$$

Here, $c(c>0)$ represents the surface stretching sheet associated parameter and $n$ is the power index associated with the surface stretching speed. $T_{w}$ and $T_{\infty}$ are the stable temperatures at the sheet and free stream temperature respectively. Moreover, the similarity variables introduced in Equation (6) may be written as

$$
\left.\begin{array}{l}
u=c x^{n} f^{\prime}(\eta) \\
\eta=y \sqrt{\frac{a(n+1)}{2 v}} x^{\frac{n-1}{2}} \\
T=\left(T_{w}-T_{\infty}\right) \theta(\eta)+T_{\infty} \\
C=\left(C_{w}-C_{\infty}\right) \phi(\eta)+C_{\infty} \\
v=-\sqrt{c v\left(\frac{n+1}{2}\right)} x^{\frac{n-1}{2}}\left[f(\eta)+\frac{n-1}{n+1} \eta f^{\prime}(\eta)\right]
\end{array}\right\}
$$


The radiative heat flux $\left(q_{r}\right)$ described by Rossland approximation takes the form

$$
\frac{\partial q_{r}}{\partial y}=-\frac{16 \sigma^{*} T_{\infty}^{3}}{3 k^{*}} \frac{\partial^{2} T}{\partial y^{2}},
$$

with

$$
q_{r}=-\frac{4 \sigma^{*}}{3 k^{*}} \frac{\partial T^{4}}{\partial y} .
$$

where $\sigma^{*}$ designates the Stefan-Boltzman constant and $k^{*}$ designates the Rossland mean absorption coefficient. After employing the similarity variables, Equation (2) is identically satisfied. The rest of the Equations (3)-(5) are transformed into coupled nonlinear ordinary differential equations that take the form

$$
\begin{gathered}
\left(1+\frac{1}{\beta}\right) f^{\prime \prime \prime}+f f^{\prime \prime}-2\left(\frac{n f^{\prime 2}}{n+1}\right)-\frac{2}{n+1}(K+M) f^{\prime}=0, \\
\left(1+\frac{4}{3} R d\right) \theta^{\prime \prime}+\operatorname{Pr}\left(f \theta^{\prime}+\frac{2}{n+1} Q \theta\right)+\operatorname{Pr}\left(1+\frac{1}{\beta}\right) E c f^{\prime \prime 2}=0,
\end{gathered}
$$

and

$$
\phi^{\prime \prime}+\text { Le } f \phi^{\prime}=0 \text {. }
$$

Similarly, the boundary conditions may take the form

$$
\left.\begin{array}{l}
f(0)=0, \quad f^{\prime}(0)=1, \quad \theta(0)=1, \quad \phi(0)=1 \quad \text { at } y \rightarrow 0 \\
f^{\prime}(\infty) \rightarrow 0, \quad \theta(\infty) \rightarrow 0, \quad \phi(\infty) \rightarrow 0 \quad \text { as } y \rightarrow \infty
\end{array}\right\}
$$

where $\operatorname{Pr}$ symbolize the Prandtl Number, $Q>0$ and $Q<0$ represent the heat source and the heat sink, $R d$ is the radiation parameter, $M$ is the magnetic number, $\beta$ is the Casson fluid parameter, $K$ is the permeability parameter, $E c$ is the Eckert number and $L e$ is the Lewis number. These parameters may be expressed as

$$
\begin{aligned}
& \operatorname{Pr}=\frac{\mu c_{p}}{\kappa} \\
& Q=\frac{Q_{0} x}{u_{w}(x) \rho c_{p}} \\
& R d=\frac{4 \sigma^{*} T_{\infty}^{3}}{k k^{*}} \\
& M=\frac{\sigma B_{0}^{2}}{c \rho} \\
& \beta=\frac{\mu_{B} \sqrt{2 \pi_{c}}}{p_{y}} \\
& K=\frac{v}{c k^{*}} \\
& E c=\frac{u_{w}^{2}(x)}{c_{p}\left(T_{w}-T_{\infty}\right)} \\
& L e=\frac{v}{D_{B}}
\end{aligned}
$$

when the surface is stretched linearly (i.e. $n=1.0$ ), then by setting $K=M=0$, 
the exact solution of Equation (10) may take the form

$$
f(\eta)=\sqrt{1+\frac{1}{\beta}}\left(1-\exp \left(-\frac{\eta}{\sqrt{1+1 / \beta}}\right)\right) .
$$

Comparison between the exact and numerical solutions to the problem is shown in Figure 2. The result illustrates that there is good agreement between the exact and numerical solutions.

\section{Physical Quantities}

The physical quantities of the interest are the coefficient of skin friction $c_{f}$ and the local Nusselt number $N u_{x}$, which are described by

$$
\left.\begin{array}{l}
C_{f}=\frac{\tau_{w}}{\rho u_{w}^{2}(x)} \\
N u_{x}=\frac{x q_{w}}{k\left(T_{w}-T_{\infty}\right)}
\end{array}\right\}
$$

where $\tau_{w}$ is the skin friction from the plate and $q_{w}$ is the wall heat flux from the plate. In this problem, the wall skin friction and the heat flux are described as

$$
\left.\begin{array}{l}
\tau_{w}=\mu\left(\frac{\partial u}{\partial y}\right)_{y=0} \\
q_{w}=\left[-\left(k+\frac{16 \sigma^{*} T_{\infty}^{3}}{3 k^{*}}\right)\left(\frac{\partial T}{\partial y}\right)\right]
\end{array}\right\}
$$

and

$$
\left.\begin{array}{l}
C_{f}=\left(R e_{x}\right)^{-1 / 2}\left(1+\frac{1}{\beta}\right)\left(\frac{n+1}{2}\right)^{1 / 2} f^{\prime \prime}(0) \\
N u_{x}=-\left(R e_{x}\right)^{1 / 2}\left(\frac{n+1}{2}\right)^{1 / 2}\left(1+\frac{4}{3} R d\right) \theta^{\prime}(0)
\end{array}\right\}
$$

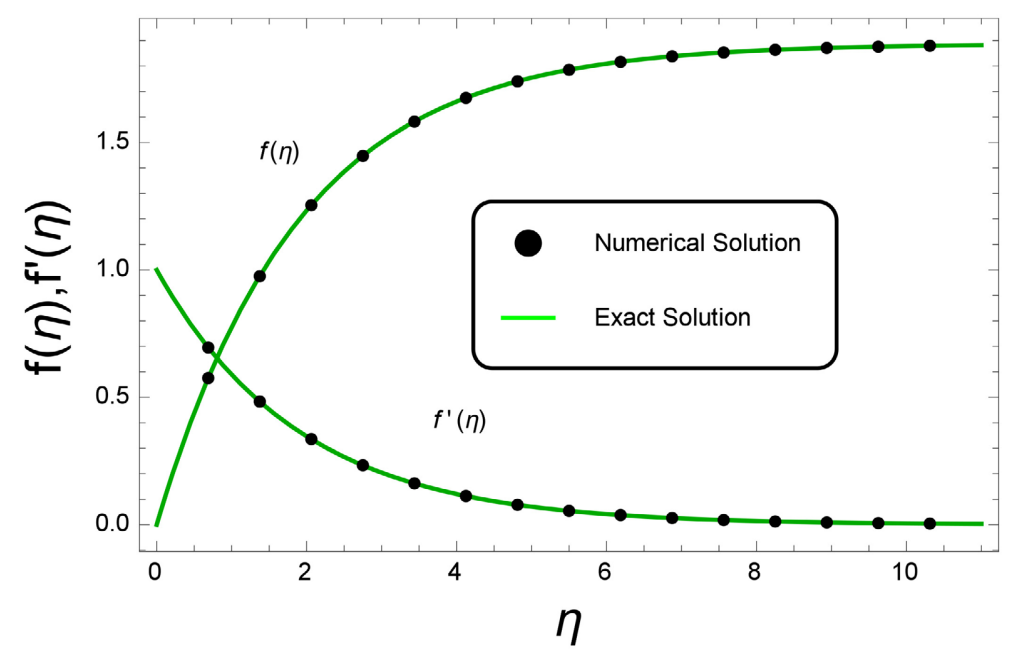

Figure 2. $f(\eta)$ and $f^{\prime}(\eta)$ for linearly stretching surface. 
Equation (18) is the dimensionless form of the skin friction $c_{f}$ and the Local Nusselt number $N u_{x}$, where $R e_{x}=U_{w} x / v$ is the local Reynolds number.

\section{Numerical Solution}

In this work, the set of Equations (10) and (11) subject to the conditions described in Equation (13) has been computed using fourth-order Runge-Kutta method with shooting technique by the use of computational software; MATHEMATICA. The values of $f(0)$ and $f^{\prime}(0)$ are known, while we take an arbitrary value for $f^{\prime \prime}(0)$, then improve it by an iterative process till the boundary conditions for large values of $\eta$ satisfied completely. The iterative process to get an appropriate value of $f^{\prime \prime}(0)$ is called the shooting technique. In order to understand the physical overview of the problem, an arrangement of figures has been displayed in the next section.

\section{Results and Discussion}

This section provides a physical overview of the emerging flow parameters on velocity and energy profiles.

\subsection{Velocity Profile}

Figure 3 illustrates the variation in velocity profile with an increase in values of $\beta$ against $\eta$, which demonstrates that for higher values of $\beta$, the fluid velocity reduces effectively. This is because, by increasing $\beta$, we are increasing the viscous forces, which slows down the flow speed. Similarly, Figure 4 illustrates the behavior of the nonlinear stretching parameter. It is observed that for larger stretching, the flow velocity increases significantly, and large momentum boundary layer thickness is witnessed.

The influence of the magnetic number is shown in Figure 5, which clearly demonstrates that the fluid velocity decreases as $M$ increases. This occurs due to the strong dominance of the viscous forces over electromagnetic forces. Figure 6

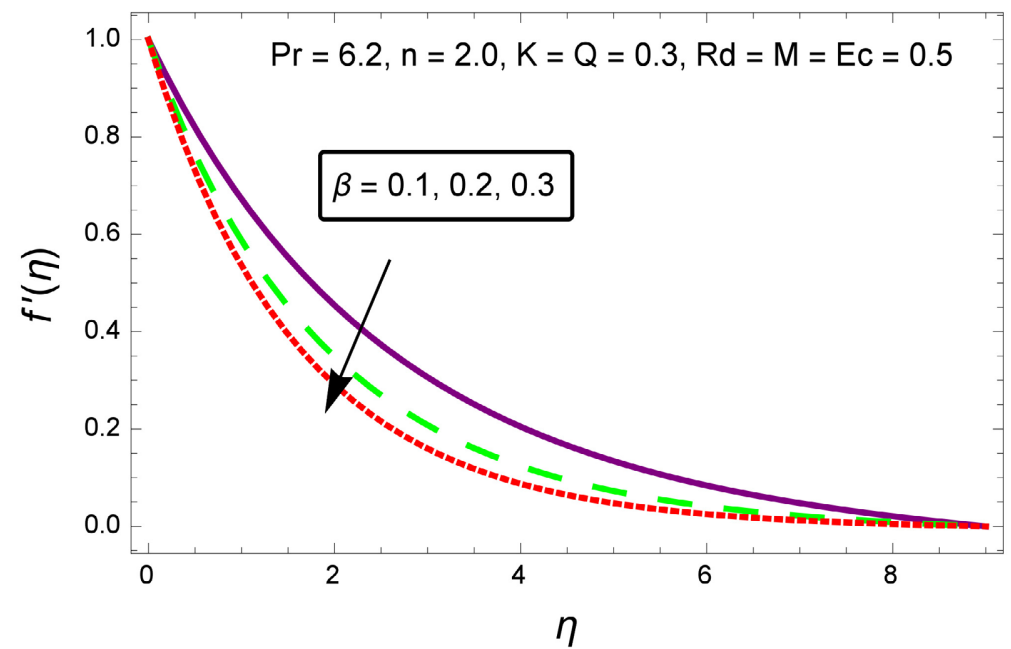

Figure 3. Variation of Casson's parameter in velocity profile. 


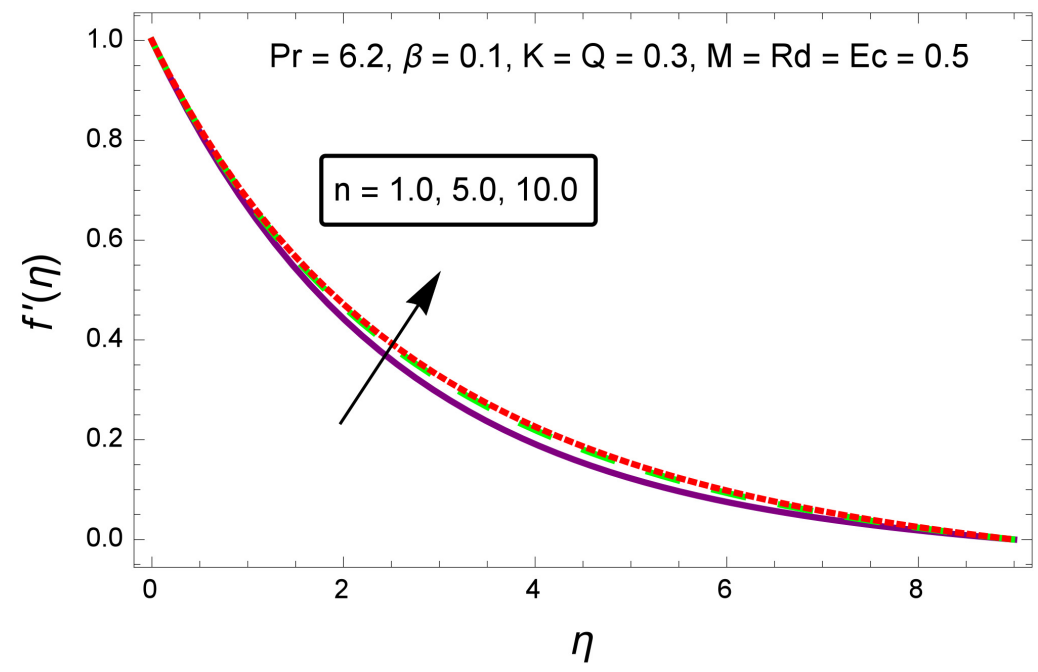

Figure 4. Variation of stretching parameter in velocity profile.

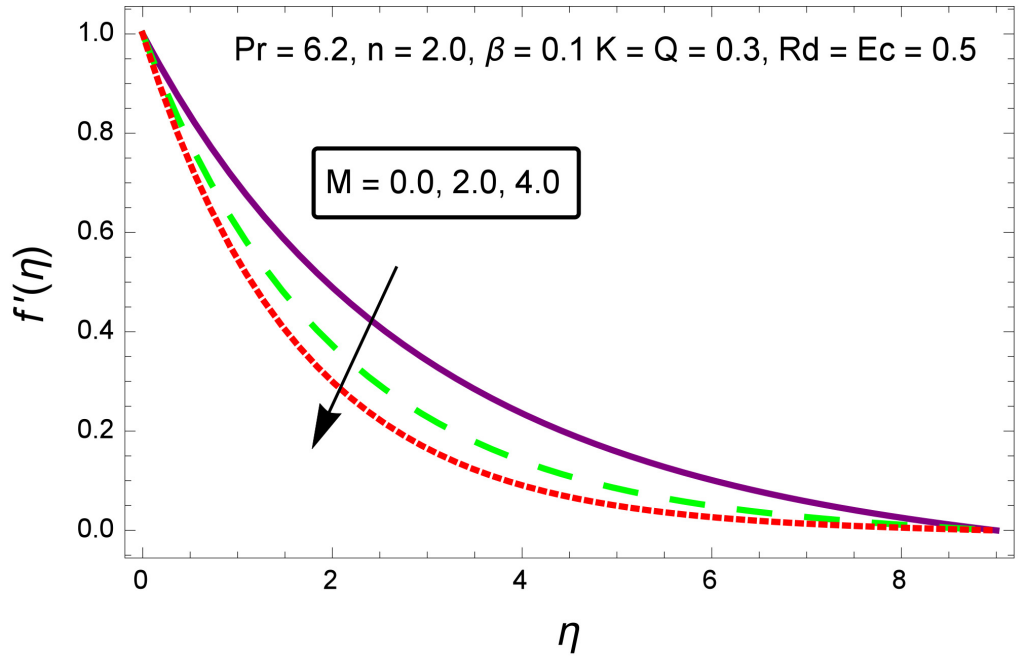

Figure 5. Variation of magnetic number in velocity profile.

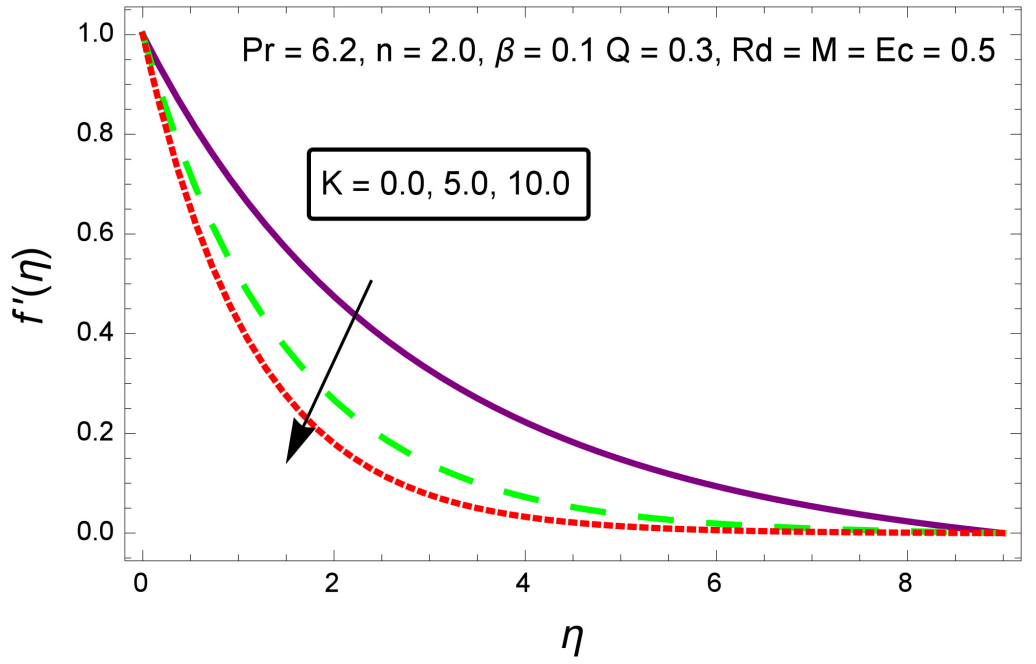

Figure 6. Variation of permeability parameter in velocity profile. 
shows the behavior of permeability parameter $K$ with variation in $f^{\prime}$. The results conclude that by accelerating $K$, the fluid velocity reduces effectively.

\subsection{Temperature Profile}

Figure 7 depicts temperature profile variation with an increasing magnitude of $\beta$. It's been observed that with increased values of $\beta$, the temperature profile increases significantly. This is because, by increasing $\beta$, we are basically boosting the viscous forces, which retards the flow velocity. Hence, an increase in flow temperature occurs. Figure 8 demonstrates the variation in the temperature field with an enhancing value of $E c$. It is observed that by increasing values of $E c$, the temperature distribution boost dramatically, whereas Figure 9 shows that by enlarging $P r$, the flow temperature decreases effectively. Similarly, the energy and thermal boundary layer thickness reduce with an increase in Pr. Physically, it

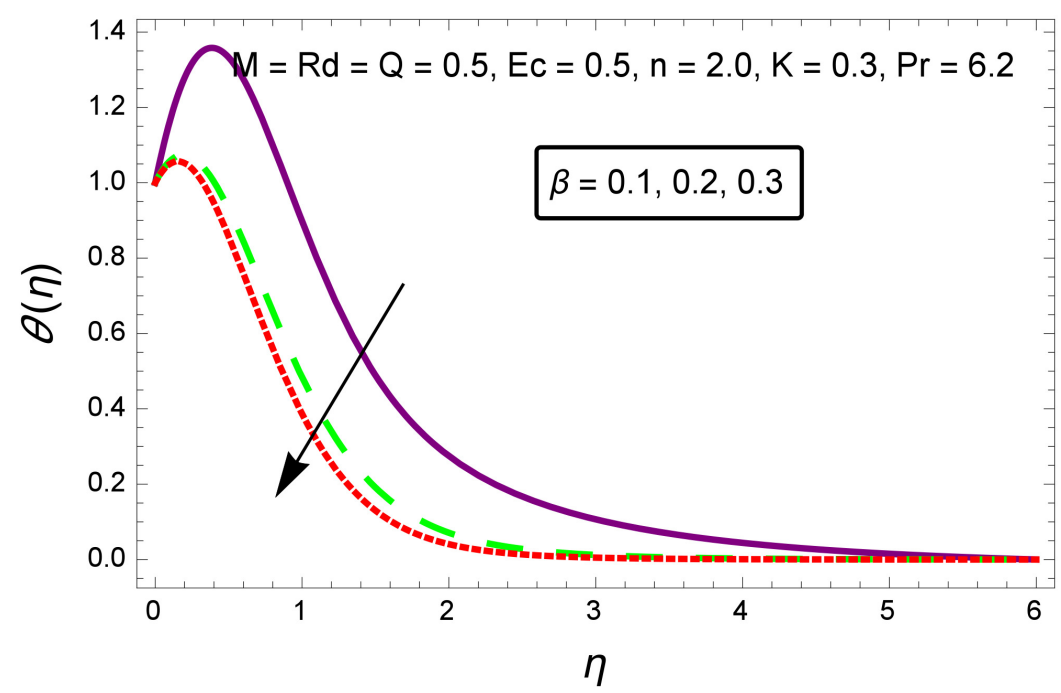

Figure 7. Variation of Casson's parameter in temperature profile.

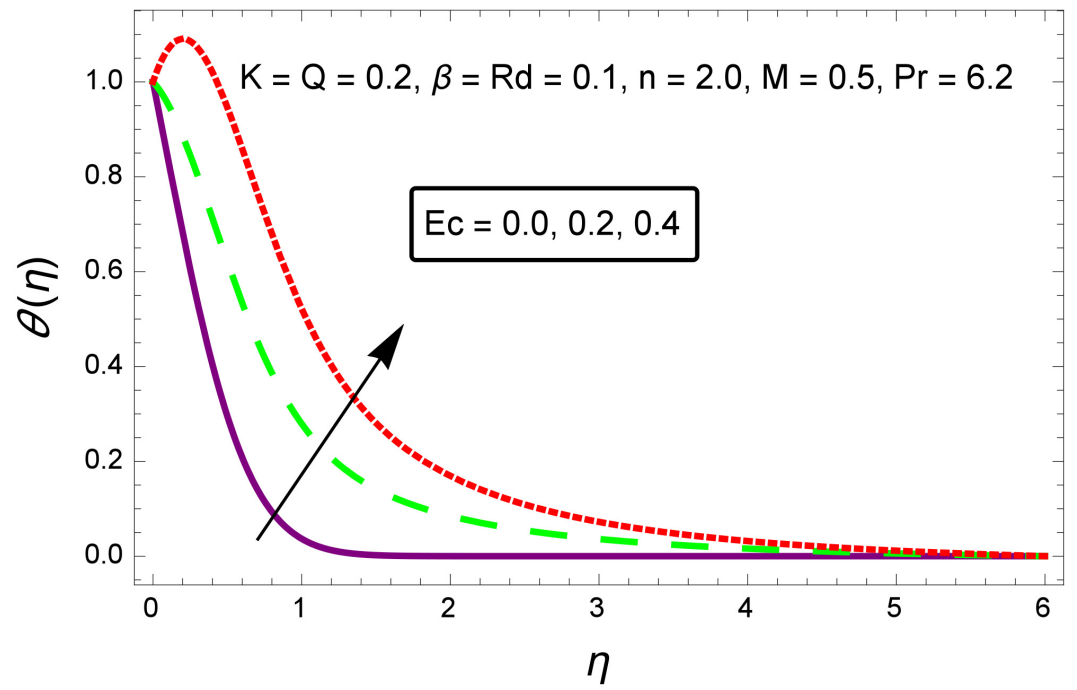

Figure 8. Variation of Eckert number in temperature profile. 


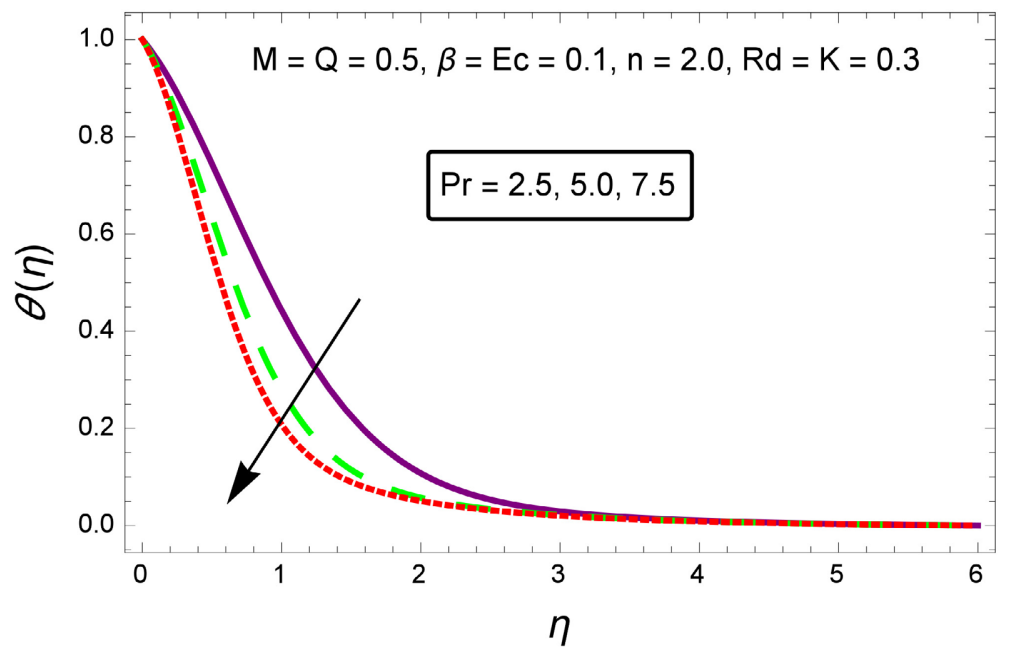

Figure 9. Variation of Prandtl number in temperature profile.

explains that an increment in the Prandtl number means a boost in the fluid viscosity, which causes a reduction in the temperature distribution.

The effect of radiation parameter on energy profile is plotted in Figure 10. The plots conclude that the acceleration in radiation parameter leads to a boost in the fluid energy level as well as the thickness of the thermal boundary layer. Moreover, Figure 11 and Figure 12 illustrate the impact of the heat source parameter on $\theta(\eta)$. In the case of Figure 11, it is acknowledged that by increase in magnitude $Q>0$, the fluid temperature increases significantly. Besides this, in the case of Figure 12, it is examined that by reducing the magnitude of $Q<0$, the temperature profile decreases effectively. Basically, in the first case, the heat source can provide additional heat to the sheet which correspondingly enhances the fluid energy, whereas in the second one, the heat sink reduces the heat generation from the stretching surface, which consequently reduces the flow temperature.

\subsection{Concentration Profile}

The variation of Casson parameter on the fluid concentration is shown in Figure 13 and it is observed that the flow concentration accelerates well with an increment in the Casson parameter. The influence of non-dimensional Lewis parameter is plotted in Figure 14 and acknowledges that Lewis number retards the fluid concentration effectively. In the same manner, the distribution of magnetic parameter on fluid concentration is displayed in Figure 15, which depicts that the concentration of fluid increases effectively due to an increase in magnetic effects.

\subsection{Wall Skin Friction and Nusselt Number}

Table 1 shows the numerical computation of the wall skin friction and Nusselt number variation. The magnitude of the wall skin friction coefficient and the local Nusselt number decreases as the Casson parameter increases. Furthermore, 


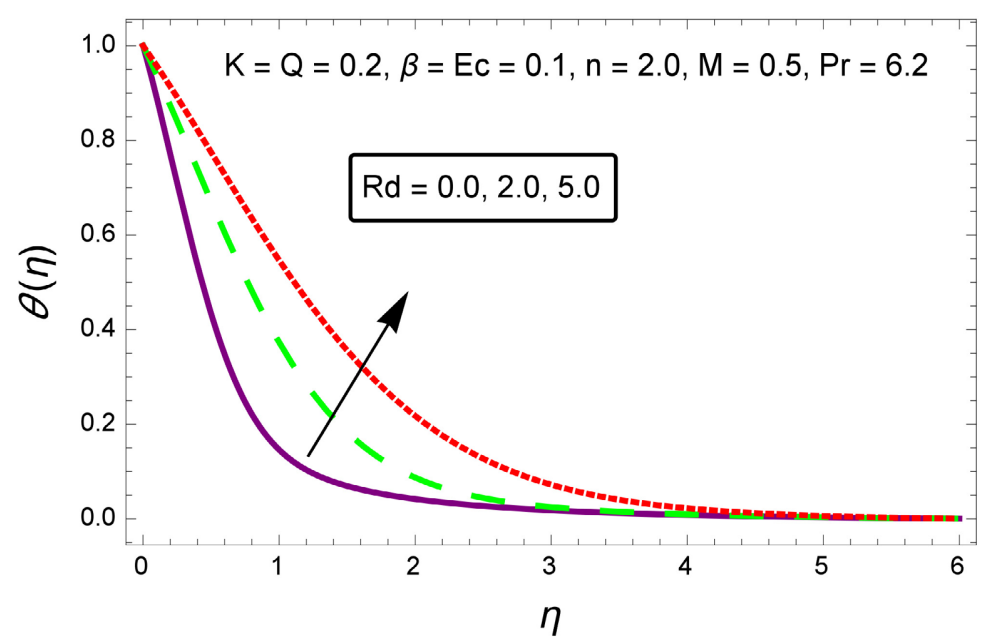

Figure 10. Variation of radiation parameter in temperature profile.

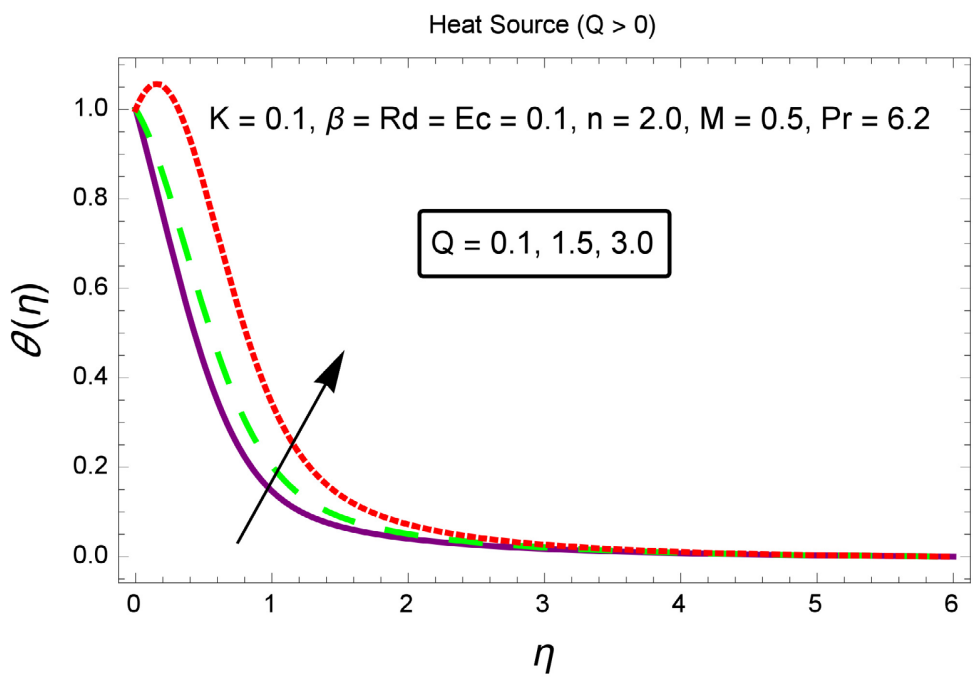

Figure 11. Variation of heat source parameter in temperature profile.

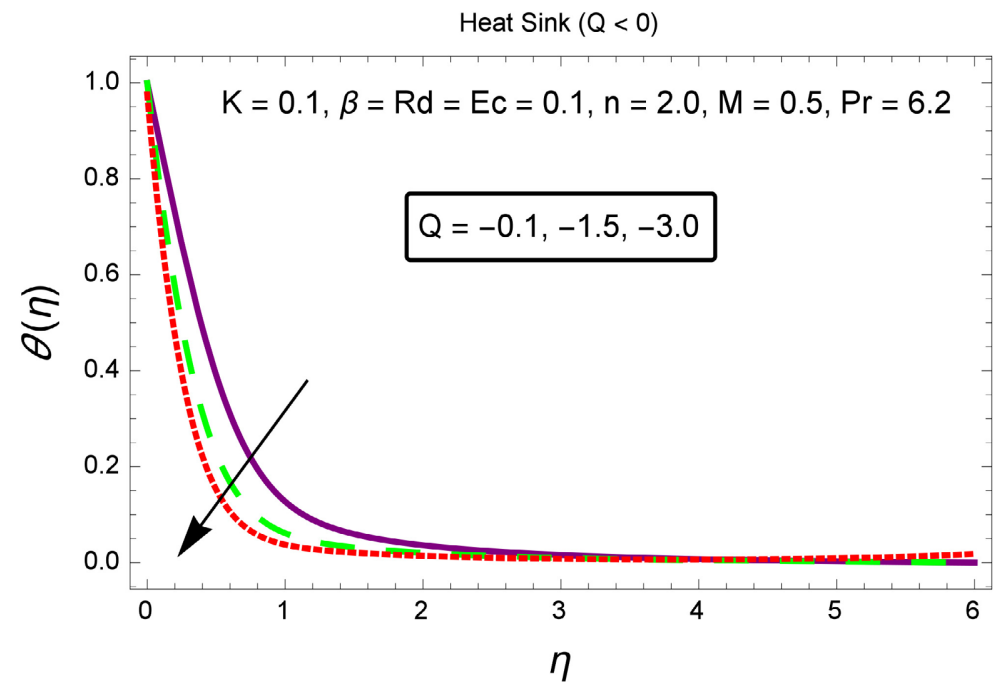

Figure 12. Variation of heat sink parameter in temperature profile. 


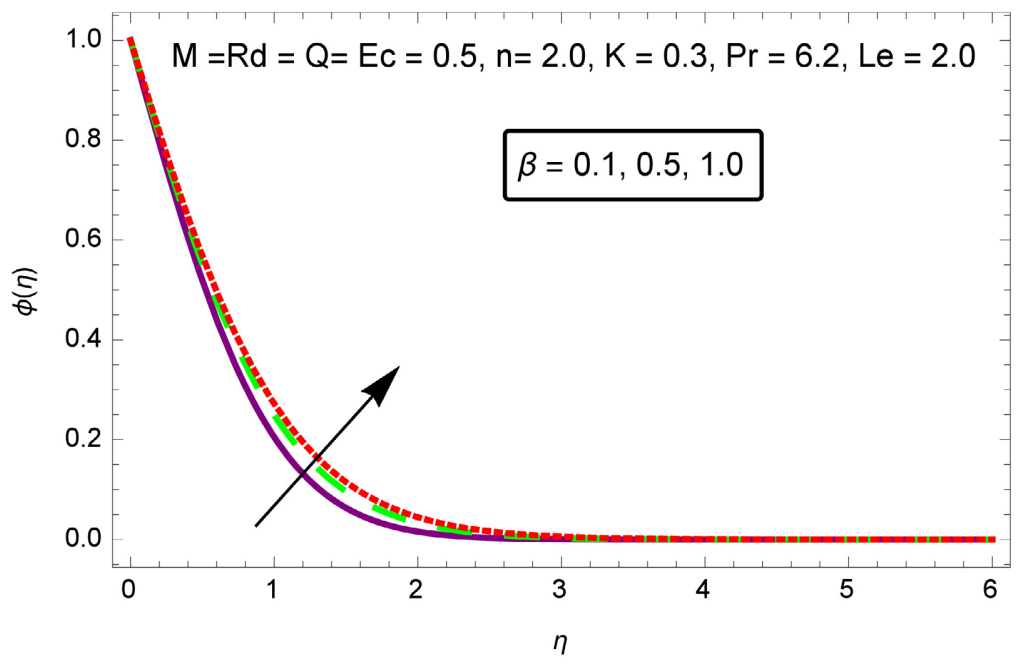

Figure 13. Variation of Casson parameter in concentration profile.

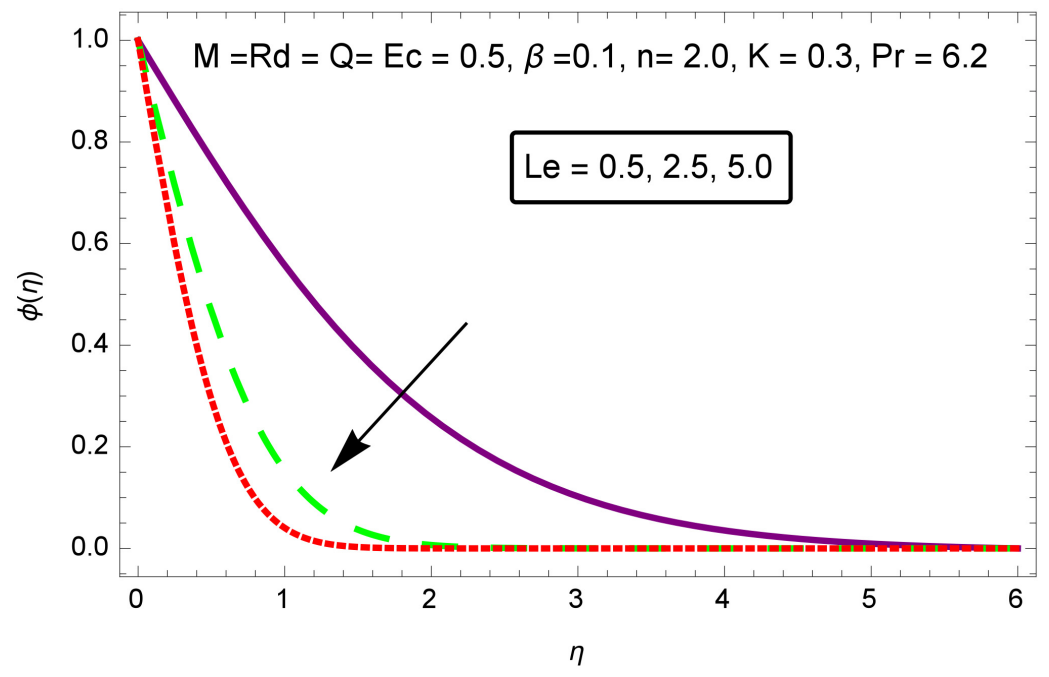

Figure 14. Variation of Lewis parameter in concentration profile.

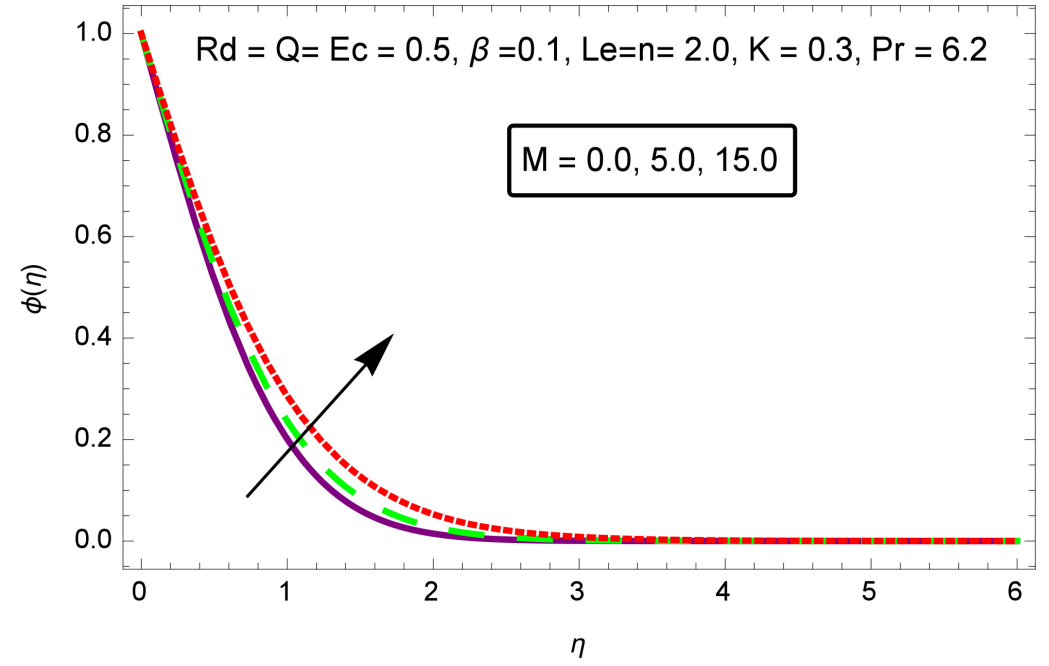

Figure 15. Variation of magnetic number in concentration profile. 
Table 1. Variations of skin friction coefficient $-f^{\prime \prime}(0)$ and $-\theta^{\prime}(0)$ for various values of dimensionless governing parameters.

\begin{tabular}{cccccccccc}
\hline$\beta$ & $M$ & $K$ & $n$ & $Q$ & $R d$ & $P r$ & $E c$ & $-f^{\prime \prime}(0)$ & $-\theta^{\prime}(0)$ \\
\hline 0.1 & 0.5 & 0.5 & 2.0 & 0.3 & 0.1 & 6.2 & 0.5 & 2.92568 & 1.07127 \\
0.5 & -- & -- & -- & -- & -- & -- & -- & 2.70282 & 0.85123 \\
1.0 & -- & -- & -- & -- & -- & -- & -- & 2.45380 & 0.68488 \\
0.5 & 1.0 & -- & -- & -- & -- & -- & -- & 3.11091 & 1.00157 \\
-- & 5.0 & -- & -- & -- & -- & -- & -- & 2.55136 & 0.85123 \\
-- & 9.0 & -- & -- & -- & -- & -- & -- & 2.36889 & 0.69605 \\
-- & 0.5 & 0.0 & -- & -- & -- & -- & -- & 2.49683 & 0.99336 \\
-- & -- & 1.0 & -- & -- & -- & -- & -- & 2.70282 & 0.85123 \\
-- & -- & 4.0 & -- & -- & -- & -- & -- & 3.21406 & 0.50748 \\
\hline
\end{tabular}

as the magnetic parameter is increased, the magnitude of the wall skin friction coefficient and the local Nusselt number decrease. However, the magnitude of the wall skin friction coefficient grows as permeability increases, but the local Nusselt number decreases as the magnetic parameter increases.

\section{Conclusion}

In this work, we transform the governing equations into coupled nonlinear ordinary differential equations and perform the numerical computation using the fourth order Runge-Kutta method. We examined the influence of several physical parameters, such as the Casson parameter, nonlinear stretching, heat source/sink parameter, radiation parameter, magnetic parameter, permeability parameter, Prandtl number, Eckert number, Lewis number on the flow field, concentration distribution, temperature, and represented them graphically. From the above description, the main conclusions of our work are summarized as follows:

- The flow velocity is a decreasing function of Casson and magnetic parameter.

- The temperature field is an increasing function of Eckert number and thermal radiation.

- The heat source parameter is directly proportional to the temperature field, whereas the heat sink parameter is proportional inversely.

- The magnitude of the wall skin friction coefficient grows as the permeability increases, but the local Nusselt number decreases as the magnetic parameter increases.

- The magnitude of the wall skin friction coefficient and local Nusselt number reduces with variation in Casson parameter.

- The fluid concentration increases for large values of Casson and magnetic parameters and decreases for Lewis number.

\section{Acknowledgements}

I would like to thank Ms. Yin Feng and Mr. Pir Muhammad Ismail. Both were instrumental in defining the path of my research. For this, I am extremely grateful. 


\section{Conflicts of Interest}

The authors declare no conflicts of interest regarding the publication of this paper.

\section{References}

[1] Casson, N. (1959) A Flow Equation for Pigment-Oil Suspensions of the Printing Ink Type. In: Mill, C.C., Ed., Rheology of Disperse Systems, Pergamon Press, Oxford, 84-102.

[2] Subba Rao, A., Ramachandra Prasad, V., Bhaskar Reddy, N. and Anwar Bég, O. (2015) Heat Transfer in a Casson Rheological Fluid from a Semi-Infinite Vertical Plate with Partial Slip. Heat Transfer Asian Research, 44, 272-291.

https://doi.org/10.1002/htj.21115

[3] Bird, R.B., Dai, G. and Yarusso, B.J. (1983) The Rheology and Flow of Viscoplastic Materials. Reviews in Chemical Engineering, 1, 1-70.

https://doi.org/10.1515/revce-1983-0102

[4] Mukhopadhyay, S. (2013) Casson Fluid Flow and Heat Transfer over a Nonlinearly Stretching Surface. Chinese Physics B, 22, Article ID: 074701. https://doi.org/10.1088/1674-1056/22/7/074701

[5] Animasaun, I., Adebile, E. and Fagbade, A. (2016) Casson Fluid Flow with Variable Thermo-Physical Property along Exponentially Stretching Sheet with Suction and Exponentially Decaying Internal Heat Generation Using the Homotopy Analysis Method. Journal of the Nigerian Mathematical Society, 35, 1-17. https://doi.org/10.1016/j.jnnms.2015.02.001

[6] Nawaz, M., Naz, R. and Awais, M. (2018) Magnetohydrodynamic Axisymmetric Flow of Casson Fluid with Variable Thermal Conductivity and Free Stream. Alexandria Engineering Journal, 57, 2043-2050. https://doi.org/10.1016/j.aej.2017.05.016

[7] Awais, M., Raja, M.A.Z., Awan, S.E., Shoaib, M. and Ali, H.M. (2021) Heat and Mass Transfer Phenomenon for the Dynamics of Casson Fluid through Porous Medium over Shrinking Wall Subject to Lorentz Force and Heat Source/Sink. Alexandria Engineering Journal, 60, 1355-1363. https://doi.org/10.1016/j.aej.2020.10.056

[8] Alfvén, H. (1942) Existence of Electromagnetic-Hydrodynamic Waves. Nature, 150, 405-406. https://doi.org/10.1038/150405d0

[9] Anantha Kumar, K., Sugunamma, V., and Sandeep, N. (2020) Effect of Thermal Radiation on MHD Casson Fluid Flow over an Exponentially Stretching Curved Sheet. Journal of Thermal Analysis and Calorimetry, 140, 2377-2385. https://doi.org/10.1007/s10973-019-08977-0

[10] Hayat, T., Bibi, S., Rafiq, M., Alsaedi, A. and Abbasi, F. (2016) Effect of an Inclined Magnetic Field on Peristaltic Flow of Williamson Fluid in an Inclined Channel with Convective Conditions. Journal of Magnetism and Magnetic Materials, 401, 733-745. https://doi.org/10.1016/j.jmmm.2015.10.107

[11] Hussain, S. (2017) Finite Element Solution for MHD Flow of Nanofluids with Heat and Mass Transfer through a Porous Media with Thermal Radiation, Viscous Dissipation and Chemical Reaction Effects. Advances in Applied Mathematics and Mechanics, 9, 904-923. https://doi.org/10.4208/aamm.2014.m793

[12] Dero, S., Rohni, A.M. and Saaban, A. (2019). MHD Micropolar Nanofluid Flow over an Exponentially Stretching/Shrinking Surface: Triple Solutions. Journal of Advanced Research in Fluid Mechanics and Thermal Sciences, 56, 165-174

[13] Sumalatha, C., Bandari, S., et al. (2015) Effects of Radiations and Heat Source/Sink 
on a Casson Fluid Flow over Nonlinear Stretching Sheet. World Journal of Mechanics, 5, 257-265. https://doi.org/10.4236/wjm.2015.512024

[14] Rudraswamy, N., Kumar, K.G., Gireesha, B. and Gorla, R. (2016) Soret and Dufour Effects in Three-Dimensional Flow of Jeffery Nanofluid in the Presence of Nonlinear Thermal Radiation. Journal of Nanoengineering and Nanomanufacturing, 6 , 278-287. https://doi.org/10.1166/jnan.2016.1293

[15] Kumar, K.G., Rudraswamy, N., Gireesha, B. and Krishnamurthy, M. (2017) Influence of Nonlinear Thermal Radiation and Viscous Dissipation on Three-Dimensional Flow of Jeffrey Nano Fluid over a Stretching Sheet in the Presence of Joule Heating. Nonlinear Engineering, 6, 207-219. https://doi.org/10.1515/nleng-2017-0014

[16] Rudraswamy, N., Kumar, K.G., Gireesha, B., Gorla, R., et al. (2017) Combined Effect of Joule Heating and Viscous Dissipation on MHD Three Dimensional Flow of a Jeffrey Nanofluid. Journal of Nanofluids, 6, 300-310.

https://doi.org/10.1166/jon.2017.1329

[17] Sheikholeslami, M., Hayat, T. and Alsaedi, A. (2017) Numerical Study for External Magnetic Source Influence on Water Based Nanofluid Convective Heat Transfer. International Journal of Heat and Mass Transfer, 106, 745-755. https://doi.org/10.1016/j.ijheatmasstransfer.2016.09.077

[18] Sheikholeslami, M. (2017) Magnetic Field Influence on Nanofluid Thermal Radiation in a Cavity with Tilted Elliptic Inner Cylinder. Journal of Molecular Liquids, 229, 137-147. https://doi.org/10.1016/j.molliq.2016.12.024

[19] Waqas, M., Khan, M.I., Hayat, T., Alsaedi, A. and Khan, M.I. (2017) Nonlinear Thermal Radiation in Flow Induced by a Slendering Surface Accounting Thermophoresis and Brownian Diffusion. The European Physical Journal Plus, 132, 1-13. https://doi.org/10.1140/epjp/i2017-11555-0

[20] Kumar, K.G., Rudraswamy, N., Gireesha, B. and Manjunatha, S. (2017) Non Linear Thermal Radiation Effect on Williamson Fluid with Particle-Liquid Suspension past a Stretching Surface. Results in Physics, 7, 3196-3202.

https://doi.org/10.1016/j.rinp.2017.08.027

[21] Ellahi, R., Alamri, S.Z., Basit, A. and Majeed, A. (2018) Effects of MHD and Slip on Heat Transfer Boundary Layer Flow over a Moving Plate Based on Specific Entropy Generation. Journal of Taibah University for Science, 12, 476-482. https://doi.org/10.1080/16583655.2018.1483795

[22] Hussain, F., Ellahi, R. and Zeeshan, A. (2018) Mathematical Models of electroMagnetohydrodynamic Multiphase Flows Synthesis with Nano-Sized Hafnium Particles. Applied Sciences, 8, Article No. 275. https://doi.org/10.3390/app8020275

[23] Kumar, K.A., Reddy, J.R., Sugunamma, V. and Sandeep, N. (2019) Simultaneous Solutions for MHD Flow of Williamson Fluid over a Curved Sheet with Nonuniform Heat Source/Sink. Heat Transfer Research, 50, 581-603. https://doi.org/10.1615/HeatTransRes.2018025939

[24] Lund, L.A., Omar, Z., Khan, I., Baleanu, D. and Nisar, K.S. (2020) Dual Similarity Solutions of MHD Stagnation Point Flow of Casson Fluid with Effect of Thermal Radiation and Viscous Dissipation: Stability Analysis. Scientific Reports, 10, 1-13. https://doi.org/10.1038/s41598-020-72266-2

[25] Mabood, F. and Das, K. (2019) Outlining the Impact of Melting on MHD Casson Fluid Flow Past a Stretching Sheet in a Porous Medium with Radiation. Heliyon, 5, e01216. https://doi.org/10.1016/j.heliyon.2019.e01216

[26] Ali Lund, L., Omar, Z., Khan, I., Raza, J., Bakouri, M. and Tlili, I. (2019) Stability Analysis of Darcy-Forchheimer Flow of Casson Type Nanofluid over an Exponen- 
tial Sheet: Investigation of critical points. Symmetry, 11, Article No. 412.

https://doi.org/10.3390/sym11030412

[27] Awais, M., Hayat, T., Irum, S. and Saleem, S. (2015) Dual Solutions for Nonlinear Flow Using Lie Group Analysis. PLoS ONE, 10, e0142732.

https://doi.org/10.1371/journal.pone.0142732

[28] Renard, N. and Deck, S. (2016) A Theoretical Decomposition of Mean Skin Friction Generation into Physical Phenomena across the Boundary Layer. Journal of Fluid Mechanics, 790, 339-367. https://doi.org/10.1017/jfm.2016.12

[29] Awais, M., Malik, M., Hussain, A., Salahuddin, T., et al. (2017) A Computational Analysis Subject to Thermophysical Aspects of Sisko Fluid Flow over a Cylindrical Surface. The European Physical Journal Plus, 132, 1-16.

https://doi.org/10.1140/epjp/i2017-11645-y 